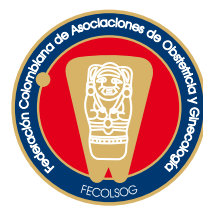

INVESTIGACIÓN ORIGINAL

\title{
PRESENCIA DE ALTERACIONES HISTOPATOLÓGICAS EN VELLOSIDADES PLACENTARIAS NORMALES EN MARACAY (VENEZUELA)
}

\section{The presence of hystopathological alterations in normal placental villi. in Maracay, Venezuela}

Olivar C. Castejón-S. *, Ángela J. López-G.**, Luis M. Pérez-Ybarra***, Óliver C. Castejón-M.****

Recibido: febrero 10/09 - Aceptado: agosto 10/09

\section{RESUMEN}

Objetivo: determinar la proporción de las alteraciones histopatológicas en las vellosidades y en el espacio intervelloso obtenida de cuatro placentas normales del último trimestre del embarazo.

Metodología: por medio de la microscopía de luz y la tinción de hematoxilina y eosina (H\&E) se identificaron, en 25 láminas de 5 regiones de la placenta, 6 variables cuantitativas (p.ej. inmadurez vellosa, nódulos sincitiales, cambios fibrinoides, edema velloso, fibrosis estromal y calcificación) y 9 variables cualitativas (p.ej. depósitos de fibrina, trombosis intervellosa, infartos, trombosis vascular, cambios en la pared del vaso, calcificación intraluminal, congestión vascular, inflamación y hemorragia). Los

* Profesor Titular en Biología Celular. Director del CIADANA.

Laboratorio de Microscopía Electrónica, Facultad de Ciencias de la Salud de la Universidad de Carabobo - Núcleo Aragua. Centro de Investigación y Análisis Docente Asistencial del Núcleo Aragua (CIADANA). Maracay, Edo. Aragua. Venezuela. Apdo. 4944. Correo electrónico: olivar.ciadanauc@gmail.com. Tel: 0243-2713305, Ext. 102. Fax: 0243-2713312.

** Magister Scientiarum en Morfología. Profesora Agregado de Morfología, Departamento de Ciencias Morfofisiológicas, Escuela de Bioanálisis, Facultad de Ciencias de la Salud, Universidad de Carabobo - Núcleo Aragua. Venezuela.

*** Profesor Agregado de Matemática, Departamento de Ciencias Básicas, Escuela de Bioanálisis, Facultad de Ciencias de la Salud, Universidad de Carabobo - Núcleo Aragua. Venezuela.

**** Estudiante de 5to año, Escuela de Medicina, Facultad de Ciencias de la Salud, Núcleo Aragua, Pasante del CIADANA. Venezuela. resultados de las variables cuantitativas se analizaron utilizando el Análisis de Varianza (ANAVAR) de 2 vías con submuestreo y el test de Tukey. En contraste, para las variables cualitativas se aplicó la prueba de Kruskal Wallis y se estimó el porcentaje de positividad según las regiones. Dichos análisis se realizaron por medio del software Statistix ${ }^{\circledR} 8.0 \mathrm{y}$ SAS $^{\circledR} 9.0$ para Windows ${ }^{\circledR}$.

Resultados: no se presentaron diferencias significativas $(\mathrm{p}<0,05)$ entre las placentas más no entre las regiones. Del mismo modo, no se observó trombosis vascular, daño de la pared del vaso, congestión vascular, calcificación intraluminal, inflamación ni hemorragia.

Conclusión: las vellosidades analizadas se mostraron homogéneas dentro de cada placenta pero no entre las mismas, indicando una variabilidad que etiológicamente podría explicarse por factores genéticos y ambientales de cuya interacción resultarían las diferencias individuales para cada placenta.

Palabras clave: alteraciones histopatológicas, vellosidades placentarias, placentas normales.

\section{SUMMARY}

Objective: determining the percentage of histopathological changes in chorionic villi and intervillous space in four placentas obtained from normal women's pregnancies at term. 
Methodology: six quantitative variables (i.e. immaturity, syncytial knots, fibrinoid change, oedema, fibrosis and calcification) and nine qualitative variables (i.e. fibrin deposition, intervillous fibrin, infarction, thrombosis, changes in vessel walls, intraluminal calcification, vascular congestion, inflammation and haemorrhage) were indentified on 25 slides covering 5 placental regions using light microscopy and H\&E staining. Quantitative variable results were analysed using two-way variance analysis with sub-sampling and Tukey's test; qualitative variables (the percentage of positive regions) were analysed by Kruskal-Wallis test. The software used was Statistix ${ }^{\circledR} 8.0$ and SAS $^{\circledR} 9.0$ for Windows ${ }^{\circledR}$.

Results: there were significant differences $(\mathrm{p}<0.05)$ between placenta (but not between regions) regarding syncytial knots, fibrinoid change, oedema, fibrosis and calcification. Vascular thrombosis, damage to vessel walls, vascular congestion, intraluminal calcification, inflammation and/or haemorrhage were not observed.

Conclusion: the population of villi analysed was homogeneous in each placenta but not amongst them, thereby indicating variability which could be etiologically explained by genetic and environmental factors whose interaction may have resulted in the individual differences observed for each placenta.

Key words: histopathological change, chorionic villi, normal placenta.

\section{INTRODUCCIÓN}

Existen cinco tipos de vellosidades placentarias: mesenquimáticas, intermedias inmaduras, troncales, intermedias maduras y terminales, las cuales difieren en calibre, en la estructura del estroma, en la morfología y en el número de los vasos sanguíneos. Por otra parte, los espacios intervellosos que separan a las vellosidades entre sí, son muy variables y pueden estar ocupados por un material eosinófilo llamado fibrinoide. ${ }^{1}$
Además, algunos investigadores afirman que las vellosidades placentarias pueden presentar alteraciones en su morfología durante el curso de la gestación normal. ${ }^{2}$ La gran mayoría de estudios realizados en la vellosidad placentaria están basados en material recolectado de embarazos patológicos o con complicaciones de alto riesgo, de manera que el conocimiento general que se tiene de ésta es escaso en un embarazo normal, por lo cual se requiere su examen en éstas condiciones. ${ }^{3}$

Por lo tanto, el propósito de este estudio consiste en describir, mediante la microscopía de luz y la tinción de $H \& E$, las alteraciones de las vellosidades en placentas que proceden de embarazos normales y así, determinar la proporción de las lesiones encontradas según un protocolo de variables empleado en el diagnóstico de su estructura.

\section{MATERIALES Y MÉTODOS}

Diseño: serie de casos.

Población: se seleccionaron aquellas mujeres que se encontraban en su último trimestre de embarazo normal o aquellas con recién nacidos sanos quienes acudieron a la consulta prenatal de la Maternidad la Floresta, ubicada en Maracay (Venezuela). La primera parte del estudio consistió en entregarles el consentimiento informado para su firma de acuerdo con la declaración de Helsinki. Luego, se realizó un muestreo por conveniencia, cuyos criterios para la recolección de cuatro placentas normales se tomaron siguiendo las instrucciones señaladas en la literatura. ${ }^{4}$

Procedimiento: inmediatamente después del período de alumbramiento, se procedió a extraer las muestras realizando cuatro cortes en los cuadrantes marginales y uno en el centro para obtener así cinco piezas de cada placenta. Cada una se colocó en un envase de plástico estéril que contenía formaldehído bufferado al $10 \%$ para más tarde procesarlas con la tinción de H\&E. De cada pieza placentaria, se obtuvieron cinco láminas de $\mathrm{H} \& \mathrm{E}$ con 3 a 5 cortes seriados, para un total de 25 láminas por placenta. Las 
láminas obtenidas fueron observadas y fotografiadas usando un fotomicroscopio Standard Clínico Zeiss. Variables a medir: se analizó la inmadurez vellosa (se determinó con base en la prevalencia de vellosidades intermedias inmaduras en las cuales se presentan membranas vasculosincitiales centrales). Además, se evaluaron los nódulos sincitiales, los cambios fibrinoides, el edema velloso y la fibrosis estromal, las cuales hacen referencia a la estructura general de la vellosidad. También se buscaron depósitos de fibrina, trombosis intervellosa e infartos, todos asociados con el espacio intervelloso; y trombosis, cambios en la pared muscular del vaso, calcificación intraluminal, congestión vascular e inflamación y hemorragia, referidas a los vasos de las vellosidades. Dichas variables fueron observadas siguiendo los criterios descritos en la literatura sobre patología placentaria. ${ }^{1}$ Para las variables cuantitativas, se analizaron 100 vellosidades por cada variable (aprox. 15.000 vellosidades por placenta) con objetivo de 10X (fibrosis estromal y calcificación) y 40X (inmadurez vellosa, nódulos sincitiales, cambios fibrinoides y edema velloso). En cuanto a las variables cualitativas, se observó la totalidad de la lámina con objetivo de 10X y 40X, reportando como positivo $(+)$ si la variable estaba presente y como negativo (-) si estaba ausente. Posteriormente, se calcularon los porcentajes de positividad por región, dividiendo el total de láminas positivas entre el número de láminas obtenidas por región (5 en el presente estudio).

Los datos obtenidos para las variables cuantitativas se analizaron utilizando el Análisis de Varianza (ANAVAR) de 2 vías en donde las cinco láminas por región constituyeron el submuestreo. Además, para verificar si existían diferencias significativas tanto en las placentas como en las regiones, se utilizó la prueba de la diferencia honestamente significativa (DHS) de Siegel y Tukey. ${ }^{5}$ Asimismo, se utilizó la prueba de Kruskal-Wallis ${ }^{6}$ para verificar si existían diferencias significativas entre las regiones y placentas en estudio. Posteriormente, se llevó a cabo una prueba de comparaciones de medias de rangos no paramétrica en la que se buscaba identificar las placentas o regiones con mayor o menor porcentaje de positividad. Este análisis fue posible por medio del software Statistix ${ }^{\circledR} 8.0$ y SAS ${ }^{\circledR} 9.0$ para Windows ${ }^{\circledR}$.

\section{RESULTADOS}

No se encontraron alteraciones histopatológicas en la estructura de las vellosidades placentarias.

Inmadurez vellosa: no se observaron diferencias significativas entre las cinco regiones consideradas en el estudio $(p=0,2077)$ ni entre las cuatro placentas $(\mathrm{p}=0,3441)$. Tabla 1 .

Nódulos sincitiales: se encontraron diferencias significativas entre las placentas $(\mathrm{p}<0,0001)$ pero no entre las cinco regiones $(p=0,2731)$, lo cual sugiere que existe homogeneidad intraplacentaria además de variabilidad entre los individuos. En la tabla 2 se presentan los porcentajes de las vellosidades placentarias con nódulos sincitiales en relación con la placenta y con la región en estudio. Se concluyó que la placenta 1 mostró un mayor porcentaje de vellosidades placentarias con nódulos sincitiales $(24,72 \%)$ que el resto de las placentas.

Cambios fibrinoides: se observaron diferencias significativas entre las placentas $(\mathrm{p}<0,0001)$ pero no entre las cinco regiones consideradas $(p=0,9095)$. Tabla 3. La placenta 4 mostró un mayor porcentaje de vellosidades placentarias con cambios fibrinoides $(14,36 \%)$ que el resto de las placentas en estudio.

Edema velloso: esta alteración puede afectar a cualquier vellosidad (troncales de mediano calibre, intermedias inmaduras [vii], intermedias maduras [vim] y terminales), siendo el estroma de la misma donde se notan los cambios más acentuados. El estroma de la vellosidad se muestra conformado por una matriz en la cual se observan los miofibroblastos flotando y separados en paquetes por espacios vacíos que corresponden al fluido. De acuerdo con los resultados del estudio, no existen diferencias significativas entre las 
Tabla 1. Porcentaje de vellosidades intermedias inmaduras por placentas y regiones.

\begin{tabular}{|c|c|c|c|}
\hline PLACENTAS & $\mathbf{n}$ & \% de vii & Agrupamiento de Tukey \\
\hline 1 & 25 & $1,20 \%$ & $\mathrm{~A}$ \\
\hline 2 & 25 & $1,56 \%$ & $\mathrm{~A}$ \\
\hline 3 & 25 & $0,80 \%$ & $\mathrm{~A}$ \\
\hline 4 & 25 & $1,20 \%$ & $\mathrm{~A}$ \\
\hline REGIONES & & & \\
\hline$M_{1}$ & 20 & $1,5 \%$ & $\mathrm{~A}$ \\
\hline$M_{2}$ & 20 & $0,55 \%$ & $\mathrm{~A}$ \\
\hline$M_{3}$ & 20 & $1,25 \%$ & $\mathrm{~A}$ \\
\hline$M_{4}$ & 20 & $1,55 \%$ & $\mathrm{~A}$ \\
\hline
\end{tabular}

Número de observaciones: 100

Rango medio entre las placentas: $1,56-0,80=0,76$

Nota: grupos con igual letra no presentan diferencias significativas.

Fuente: datos de la investigación.

Tabla 2. Porcentaje de vellosidades placentarias con nódulos sincitiales por placentas y regiones.

\begin{tabular}{|c|c|c|c|}
\hline PLACENTAS & $\mathbf{n}$ & $\begin{array}{c}\text { \% de vellosidades placentarias } \\
\text { con nódulos sincitiales }\end{array}$ & Agrupamiento de Tukey \\
\hline 1 & 25 & $24,72 \%$ & A \\
\hline 2 & 25 & $11 \%$ & B \\
\hline 3 & 25 & $10,20 \%$ & B \\
\hline 4 & 25 & $7,76 \%$ & A \\
\hline REGIONES & 20 & & A \\
\hline$M_{1}$ & 20 & $13 \%$ & A \\
\hline$M_{2}$ & 20 & $16,50 \%$ & A \\
\hline$M_{4}$ & 20 & $12 \%$ & A \\
\hline
\end{tabular}

Número de observaciones: 100 .

Nota: grupos con igual letra no presentan diferencias significativas.

Fuente: datos de la investigación.

muestras $(\mathrm{p}=0,8479)$ pero sí entre las placentas analizadas $(p=0,05)$. Tabla 4 .

Fibrosis estromal: no se encontraron diferencias significativas para las cinco regiones consideradas $(p=0,0617)$. Sin embargo, sí se observaron diferencias entre las cuatro placentas analizadas $(p=0,0069)$.
Tabla 5. El estroma de las vellosidades placentarias de las cuatro placentas normales no mostró ningún signo indicativo de inflamación ni de hemorragia. Por otra parte, los vasos sanguíneos o capilares vellosos presentes en el estroma de la vellosidad no presentaron alteraciones en su estructura tales como trombosis, 
Tabla 3. Porcentaje de vellosidades placentarias con cambios fibrinoides por placentas y regiones.

\begin{tabular}{|c|c|c|c|}
\hline PLACENTAS & $\mathbf{n}$ & $\begin{array}{c}\text { \% de vellosidades placentarias } \\
\text { con cambios fibrinoides }\end{array}$ & Agrupamiento de Tukey \\
\hline 1 & 25 & $9,48 \%$ & $\mathrm{~B}$ \\
\hline 2 & 25 & $10,12 \%$ & $\mathrm{~B}$ \\
\hline 3 & 25 & $8,04 \%$ & $\mathrm{~B}$ \\
\hline 4 & 25 & $14,36 \%$ & $\mathrm{~A}$ \\
\hline REGIONES & & & \\
\hline $\mathrm{M}_{1}$ & 20 & $10,80 \%$ & $\mathrm{~A}$ \\
\hline $\mathrm{M}_{2}$ & 20 & $10,80 \%$ & $\mathrm{~A}$ \\
\hline $\mathrm{M}_{3}$ & 20 & $10,35 \%$ & $\mathrm{~A}$ \\
\hline $\mathrm{M}_{4}$ & 20 & $9,75 \%$ & $\mathrm{~A}$ \\
\hline $\mathrm{PC}$ & 20 & $10,80 \%$ & $\mathrm{~A}$ \\
\hline
\end{tabular}

Número de observaciones: 100 .

Nota: grupos con igual letra no presentan diferencias significativas.

Fuente: datos de la investigación.

\section{Tabla 4. Porcentaje de vellosidades placentarias con edema por placentas y regiones.}

\begin{tabular}{|c|c|c|c|}
\hline PLACENTAS & $\mathbf{n}$ & $\begin{array}{c}\text { \% de vellosidades placentarias } \\
\text { con edema }\end{array}$ & Agrupamiento de Tukey \\
\hline 1 & 25 & $3,0 \%$ & $\mathrm{~A}$ \\
\hline 2 & 25 & $1,8 \%$ & $\mathrm{AB}$ \\
\hline 3 & 25 & $0,16 \%$ & $\mathrm{~B}$ \\
\hline 4 & 25 & $1,32 \%$ & $\mathrm{AB}$ \\
\hline REGIONES & & & \\
\hline $\mathrm{M}_{1}$ & 20 & $1,95 \%$ & $\mathrm{~A}$ \\
\hline $\mathrm{M}_{2}$ & 20 & $2,15 \%$ & $\mathrm{~A}$ \\
\hline $\mathrm{M}_{3}$ & 20 & $1,10 \%$ & $\mathrm{~A}$ \\
\hline $\mathrm{M}_{4}$ & 20 & $1,05 \%$ & $\mathrm{~A}$ \\
\hline $\mathrm{PC}$ & 20 & $1,65 \%$ & $\mathrm{~A}$ \\
\hline
\end{tabular}

Número de observaciones: 100 .

Nota: grupos con igual letra no presentan diferencias significativas.

Fuente: datos de la investigación.

cambios en la pared muscular del vaso, congestión vascular y calcificación intraluminal.

Calcificación: no se presentaron diferencias significativas entre las cinco regiones consideradas $(\mathrm{p}=0,1222)$ pero sí entre las placentas analizadas $(\mathrm{p}=0,0080)$. Tabla 6 .
Alteraciones histopatológicas en el espacio intervelloso de las placentas normales: no se observaron diferencias significativas ni entre las cinco regiones, ni entre las cuatro placentas analizadas para los depósitos de fibrina, trombosis intervellosa ni infartos. Tabla 7. 
Tabla 5. Porcentaje de vellosidades placentarias con fibrosis estromal por placentas y regiones.

\begin{tabular}{|c|c|c|c|}
\hline PLACENTAS & $\mathbf{n}$ & $\begin{array}{c}\text { \% de vellosidades placentarias } \\
\text { con edema }\end{array}$ & Agrupamiento de Tukey \\
\hline 1 & 25 & $3,28 \%$ & $\mathrm{~A}$ \\
\hline 2 & 25 & $3,12 \%$ & $\mathrm{~A}$ \\
\hline 3 & 25 & $1,92 \%$ & $\mathrm{AB}$ \\
\hline 4 & 25 & $0,92 \%$ & $\mathrm{~B}$ \\
\hline REGIONES & & & \\
\hline $\mathrm{M}_{1}$ & 20 & $1,75 \%$ & $\mathrm{~A}$ \\
\hline $\mathrm{M}_{2}$ & 20 & $3,40 \%$ & $\mathrm{~A}$ \\
\hline $\mathrm{M}_{3}$ & 20 & $3,20 \%$ & $\mathrm{~A}$ \\
\hline $\mathrm{M}_{4}$ & 20 & $1,60 \%$ & $\mathrm{~A}$ \\
\hline $\mathrm{PC}$ & 20 & $1,60 \%$ & $\mathrm{~A}$ \\
\hline
\end{tabular}

Número de observaciones: 100 .

Rango medio entre las placentas: 3,28-0,92 $=2,36$.

Nota: grupos con igual letra no presentan diferencias significativas.

Fuente: datos de la investigación.

Tabla 6. Porcentaje de vellosidades placentarias con depósitos de calcio por placentas y regiones.

\begin{tabular}{|c|c|c|c|}
\hline PLACENTAS & $\mathbf{n}$ & $\begin{array}{c}\text { \% de vellosidades placentarias } \\
\text { con depósitos de calcio }\end{array}$ & Agrupamiento de Tukey \\
\hline 1 & 25 & $1,17 \%$ & $\mathrm{~B}$ \\
\hline 2 & 25 & $1,52 \%$ & $\mathrm{~A}$ \\
\hline 3 & 25 & $1,55 \%$ & $\mathrm{~A}$ \\
\hline 4 & 25 & $1,31 \%$ & $\mathrm{AB}$ \\
\hline REGIONES & & & $\mathrm{A}$ \\
\hline$M_{1}$ & 20 & $1,48 \%$ & $\mathrm{~A}$ \\
\hline$M_{2}$ & 20 & $1,32 \%$ & $\mathrm{~A}$ \\
\hline$M_{3}$ & 20 & $1,39 \%$ & $\mathrm{~A}$ \\
\hline$M_{4}$ & 20 & $1,55 \%$ & $\mathrm{~A}$ \\
\hline
\end{tabular}

Número de observaciones: 100.

Rango medio entre las placentas: $1,55-1,17=0,38$.

Nota: grupos con igual letra no presentan diferencias significativas.

\section{DISCUSIÓN}

La placenta, en su proceso normal de maduración, sufre una serie de cambios regresivos o degenerativos (deposición fibrinoide, calcificaciones, infartos) que dentro de unos límites, pueden ser considerados como normales. ${ }^{7}$
Se supone que existe un proceso de maduración vellosa que parece estar influenciado por parámetros genéticos, metabólicos y endocrinos. El porcentaje promedio de villitis que se observó en la región central y marginal de la placenta es cercano a los presentados por Sen y colegas, ${ }^{8}$ quienes han 


\begin{tabular}{|c|c|c|c|c|c|c|c|}
\hline \multicolumn{8}{|c|}{ Tabla 7. Porcentaje de alteraciones en el espacio intervelloso de las placentas normales. } \\
\hline $\begin{array}{c}\text { Alteración } \\
\text { Histopatológica }\end{array}$ & $\begin{array}{c}\text { Placenta } \\
\text { 1 }\end{array}$ & $\begin{array}{c}\text { Placenta } \\
\text { Placenta }\end{array}$ & $\begin{array}{c}\text { Placenta } \\
\text { Valor de p }\end{array}$ & $\begin{array}{c}\text { Valor de p } \\
\text { (placentas) } \\
\text { (regiones) }\end{array}$ \\
\hline $\begin{array}{c}\text { Depósitos de } \\
\text { fibrina }\end{array}$ & $8 \%$ & $0 \%$ & $0 \%$ & $0 \%$ & $0,5732 \mathrm{~ns}$ & $0,0829 \mathrm{~ns}$ \\
\hline $\begin{array}{c}\text { Trombosis } \\
\text { intervellosa }\end{array}$ & $8 \%$ & $0 \%$ & $4 \%$ & $0 \%$ & $0,1991 \mathrm{~ns}$ & $0,2611 \mathrm{~ns}$ \\
\hline Infartos & $8 \%$ & $4 \%$ & $4 \%$ & $8 \%$ & $0,1115 \mathrm{~ns}$ & $0,8789 \mathrm{~ns}$ \\
\hline
\end{tabular}

ns: no significativa.

Fuente: datos de la investigación.

reportado valores de hasta 5\% de vellosidades intermedias inmaduras en placentas normales a término.

Igualmente, la presencia de nódulos sincitiales en las vellosidades placentarias ha sido objeto de gran controversia. Cantle y su equipo de trabajo ${ }^{9}$ afirmaron que la mayoría de los nódulos sincitiales observados en las placentas del tercer trimestre son artefactos histológicos causados por el corte tangencial de la superficie trofoblástica. Por su lado, Jones y Fox, ${ }^{10}$ con base en los cambios degenerativos, reportaron la presencia de nódulos sincitiales en una placenta normal a término entre $10 \%$ y $30 \%$ sin ningún significado patológico. No obstante, Majumdar y colaboradores ${ }^{11}$ expusieron valores mucho más bajos que oscilaban entre $6,88 \%$ y $12,96 \%$.

Asimismo, un incremento significativo de los nódulos sincitiales suele ser evidente en las placentas provenientes de pacientes con preeclampsia, lo cual podría ser consecuencia de una mala perfusión del árbol vellositario. ${ }^{12}$ Existe la posibilidad de que la placenta 1 con incremento de nódulos esté afectada por los cortes histológicos gruesos (3-5 $\mu \mathrm{m})$, lo cual ha sido descrito por Benirschke y Kaufmann, ${ }^{1}$ quienes reportaron ausencia de nódulos sincitiales al realizar cortes ultrafinos $(0,5-1 \mu \mathrm{m})$ de las piezas histológicas.

Por otra parte, otros investigadores han postulado que entre las semanas 37 y 41, los nódulos sincitiales ocupan grandes proporciones del volumen del trofoblasto contribuyendo así a la variabilidad de su espesor. Estos nódulos representarían un intento para formar puentes entre las vellosidades placentarias y podrían servir para proteger los capilares vellosos de los cambios repentinos que suceden en el espacio intervelloso durante el parto. ${ }^{10,13}$

Con respecto al fibrinoide, algunos investigadores han reportado su presencia hasta en 10\% de las vellosidades placentarias, lo cual es considerado normal. ${ }^{14}$ En esta investigación se informan valores que coinciden con lo reportado en la literatura. ${ }^{15}$ La variabilidad en los porcentajes reportados en este estudio puede explicarse por la existencia de algunas diferencias genéticas entre los individuos. Así por ejemplo, en la enfermedad trofoblástica gestacional, se ha observado en la placenta humana una fuerte deposición de fibrinoide tipo fibrina que incluye o sepulta a numerosos sectores de vellosidades coriales, lo que ha sido interpretado como una reacción inmunológica provocada por el componente paterno de la carga diploide heterocigótica 46XY, que pareciera detener la hiperplasia del trofoblasto. ${ }^{16}$

Ante ésto, algunos investigadores han postulado que es un producto de secreción normal y otros, que proviene de un proceso degenerativo, constituyendo así el tema más controversial que se haya descrito en la patología placentaria.

De otra parte, el porcentaje de edema encontrado no superó el 3\%. Esta acumulación de líquido puede ser considerada normal al compararla con lo 
reportado por Shen y colaboradores. ${ }^{17}$ Igualmente, ésto coincide con lo informado por Castejón y Molinaro, ${ }^{18}$ los cuales encontraron vellosidades con signos de edema en placentas provenientes de pacientes tanto normotensas como hipertensas. Con respecto a la variable fibrosis estromal, los porcentajes obtenidos no superaron el 4\%. Estos resultados están entre los reportados por Majumdar y su equipo de trabajo, ${ }^{11}$ quienes han encontrado un porcentaje de vellosidades fibróticas que oscila entre $2,95 \%$ y $4,6 \%$. De igual manera, dichos resultados están por debajo de los obtenidos por Sosa y demás investigadores, ${ }^{14}$ quienes han postulado que en las placentas normales a término puede presentarse hasta 10\% de vellosidades fibróticas.

Por otro lado, el no encontrar signos indicativos de villitis indicó que las pacientes no cursaron con infecciones ni complicaciones durante el embarazo. Estos resultados coinciden con los reportados por Sosa ${ }^{14}$ quien ha propuesto que para considerar un patrón de vellosidad normal, la misma no debe presentar villitis. La trombosis, los cambios en la pared muscular, la congestión y la calcificación intraluminal que fueron variables evaluadas en los vasos o capilares vellosos no se encontraron en este estudio, coincidiendo con lo reportado por Canache y Castejón, ${ }^{19}$ quienes no observaron trombosis ni cambios en la túnica muscular de los capilares vellosos observados en las placentas normales. Con respecto a las variables estudiadas en el espacio intervelloso, los depósitos de fibrina provocan la aparición de fibrosis, infartos y muerte de las vellosidades. ${ }^{11}$ En estudios recientes, se ha postulado que las placas de fibrina intervellosa, las cuales han sido observadas en $13 \%$ de las placentas normales a término, carecen de importancia clínica siempre que no exista compromiso fetal. ${ }^{20,21}$ La presencia de pequeñas placas de fibrina en el tejido placentario normal parece estar influenciada por los cambios de los factores del mecanismo de la coagulación que se pueden detectar desde el tercer mes de la gestación. ${ }^{22}$ Por su parte, la trombosis intervellosa se manifiesta por hemorragias feto-maternas provocadas por vasos mucho más pequeños que los afectados en la variable anterior. ${ }^{23}$ Estos trombos se componen de una mezcla de sangre fetal y materna; y los movimientos fetales violentos, en combinación con fetos varones de cordón umbilical muy largo, provocan estas hemorragias que se manifiestan como coágulos que desplazan los componentes del parénquima placentario. ${ }^{24}$ En cuanto a la trombosis intervellosa, se han encontrado resultados similares, según lo indicado por Becroft e investigadores, ${ }^{21}$ quienes encontraron $6 \%$ de esta variable en relación con un feto varón en placentas normales.

De otro lado, numerosos estudios han evidenciado infartos en placentas normales a término. ${ }^{11,21,25}$ Sin embargo, el porcentaje en el cual se presentan es menor si se comparan con las placentas provenientes de embarazos de alto riesgo. Las lesiones placentarias no son necesariamente causa de un resultado desfavorable pero las anormalidades severas pueden llevar a una muerte fetal, como ya se ha descrito. ${ }^{26}$

Ahora bien, el ejercicio de reconocer estos desórdenes en la placenta normal es de extraordinario valor para el entendimiento de resultados no compatibles con la vida. ${ }^{27}$ No obstante, un gran problema con la literatura relacionada con la placenta es que la mayoría de ella ha sido producida solamente en placentas anormales, de manera que para muchos rasgos no hay claridad en lo que es patológicamente anormal y lo que es normal.,26 Algunos rasgos pueden estar en los límites normales en placentas a término mientras que en etapas más tempranas pueden ser patológicos. ${ }^{28}$ Además, la evaluación de las lesiones puede volverse mucho más compleja ya que varias condiciones pueden coexistir en la misma placenta. ${ }^{3}$

En conclusión, las alteraciones referidas a las variables cuantitativas son homogéneas para la inmadurez vellosa entre las cuatro placentas y entre las regiones de la misma pero heterogénea para las restantes variables, indicando que hay homogeneidad intraplacentaria en las regiones pero con diferencias significativas entre las placentas. Las alteraciones referidas a las variables cualitativas, con respecto 
al espacio intervelloso, mostraron homogeneidad intraplacentaria e interplacentaria debido a que no existen diferencias significativas entre las regiones ni entre las placentas, sin ninguna alteración en la estructura de los vasos de la vellosidad.

\section{AGRADECIMIENTOS}

A la Coordinación Administrativa de la Facultad de Ciencias de la Salud del Núcleo Aragua por el fondo fijo institucional para el CIADANA. Al personal médico del Servicio de Ginecología y Obstetricia de la Maternidad La Floresta en Maracay Edo. Aragua y a la T.S.U. Laury R. Gutiérrez-S. por transcribir el manuscrito.

\section{REFERENCIAS}

1. Benirschke K, Kaufmann P. Pathology of the Human Placenta. 4th ed. New York: Springer-Verlag; 2000.

2. Ventolini G, Samlowski R, Hood D. Placental findings in low-risk, singleton, term pregnancies after uncomplicated deliveries. Am J Perinatol 2004;21:325-8.

3. Hargitai B, Marton T, Cox PM. Best practice No. 178: Examination of the human placenta. J Clin Pathol 2004;57:785-92.

4. Redline RW, Heller D, Keating S, Kingdom J. Placental diagnostic criteria and clinical correlation - a workshop report. Placenta 2005;26:S114-7

5. Siegel S, Tukey JW. A non parametric sum of ranks procedure for relative spread in unpaired samples. J Am Stat Assoc 1960;55:429-45.

6. Kruskal WH, Wallis WA. Use of ranks in one - criterion variance analysis. J Am Stat Assoc 1952;48:907-11.

7. Rodríguez A, López J, Sánchez F, González R, Galera H. Degeneración vacuolar de la placenta. Clin Invest Gin Obst 1985;12:190-2.

8. Sen DK, Kaufmann P, Schweikart G. Classification of human placental villi. II. Morphometry. Cell Tissue Res 1979;200:425-34.

9. Cantle SJ, Kaufmann P, Luckhardt M, Schweikhart G. Interpretation of syncytial sprouts and bridges in the human placenta. Placenta 1987;8:221-34.

10. Jones CJ, Fox H. Syncytial knots and intervillous bridges in the human placenta: an ultraestructural study. J Anat 1977;124:275-86.
11. Majumdar S, Dasgupta H, Bhattacharya K, Bhattacharya A. A study of placental in normal and hypertensive pregnancies. J Anat Soc India 2005;54:1-9.

12. Thiet MP, Suwanvanichkij V, Hasselblatt K, Yeh J. Apoptosis in human term placenta. A morphological and gene expression study. Gynecol Obstet Invest 2000;50:88-91.

13. Mayhew TM, Barker BL. Villous trophoblast: morphometric perspectives on growth, differentiation, turnover and deposition of fibrin-type fibrinoid during gestation. Placenta 2001;22:628-38.

14. Sosa A, Alonzo JF, Reigosa A. Biopsia del lecho placentario y de las vellosidades coriales en gestaciones normales y de alto riesgo. Gac Med Caracas 1995;103:358-74.

15. Bane AL, Gillan JE. Massive perivillous fibrinoid causing recurrent placental failure. BJOG 2003;110:292-5.

16. Rice LW, Genest DR, Berkowitz RS, Goldstein DP, Bernstein MR, Redline RW. Pathologic features of sharp curettings in complete Hydatidiforme mole. Predictors of persistent gestational trophoblastic disease. J Reprod Med 1999;36:17-20.

17. Shen-Schwarz S, Ruchelli E, Brown D. Villous oedema of the placenta: a clinicopathological study. Placenta 1989;10:297-307.

18. Castejón O, Molinaro M. Cambios degenerativos coriónicos y su relación con desórdenes hipertensivos en casos de desprendimiento prematuro grave de placenta normoinserta. Gac Med Caracas 2003;111:117-22.

19. Canache LA, Castejón O. Desarrollo de la vellosidad placentaria de anclaje en desórdenes hipertensivos asociados a desprendimiento prematuro grave de la placenta normoinserta. Rev Obstet Ginecol Venez 2007;67:23-30.

20. Katzman PJ, Genest DR. Maternal floor infarction and massive perivillous fibrin deposition. Histological definitions, association with intrauterine fetal growth restriction and risk of recurrence. Pediatr Dev Pathol 2002;5:159-64.

21. Becroft DM, Thompson JM, Mitchell EA. Placental infarcts, intervillous fibrin plaques, and intervillous thrombi: incidences, coocurrences, and epidemiological associations. Pediatr Dev Pathol 2004;7:26-34.

22. López-Ramírez Y, Carvajal Z, Arocha-Pinango CL. Parámetros hemostáticos en placenta de pacientes con embarazo normal y con preeclampsia severa. Invest Clin 2006;47:233-40. 
23. Sander CM, Gilliland D, Akers C, McGranth A, Bismar TA, Swart-Hills LA. Livebirths with placental hemorrhagic endovasculitis: interlesional relationships and perinatal outcomes. Arch Pathol Lab Med 2002;126:157-64.

24. Lewis S, Perrin E. Pathology of the placenta. New York: Churchill Livingstone; 1999.

25. Udainia A, Bhagwat SS, Mehta CD. Relation between placental surface area, infarction and foetal distress in pregnancy induced hypertension with its clinical relevance. J Anat Soc India 2004;53:27-30.
26. Sun CC, Revell VO, Belli AJ, Viscardi RM. Discrepancy in pathologic diagnosis of placental lesions. Arch Pathol Lab Med 2002;126:706-9.

27. Cortés H, Muñoz H. Utilidad clínica del estudio anatomopatológico de la placenta en el Hospital Universitario San Vicente de Paúl. Rev Colomb Obstet Ginecol 2007;58:60-4.

28. Viscardi RM, Sun CC. Placental lesion multiplicity: risk factor for IUGR and neonatal cranial ultrasound abnormalities. Early Hum Dev 2001;62:1-10.

Conflicto de intereses: ninguno declarado. 